\title{
Preface: recent progress and novel applications of parallel mechanisms
}

\author{
Andreas Müller • Vincenzo Parenti Castelli • \\ Tian Huang
}

Published online: 11 January 2016

(C) Springer Science+Business Media Dordrecht 2016

\begin{abstract}
After a decade of intensive research, parallel manipulators have become reality. This has been well documented by the two international workshop on "Fundamental Issues and Future Research Directions for Parallel Mechanisms and Manipulators" that were held in 2002 in Québec, Canada and in 2008 in Montpellier, France, respectively. Despite the remarkable success of parallel manipulators, several of the fundamental issues remain to be addressed, and the third international workshop that was held in Tianjin, China on July 7-8, 2014, highlighted them clearly. This issue comprises selected papers from this workshop that brought together experts in this area from around the globe. Besides disseminating most recent research progress the workshop served as a platform to
\end{abstract}

identify the most promising future research directions for parallel manipulators as well as open theoretical problems that must be solved in order to allow for their industrial use.

Of the 53 accepted submissions that were presented at the workshop, 9 extended revised papers were selected for inclusion in this special issue. These papers cover a wide spectrum of topics ranging from novel designs for special applications, elastodynamic considerations, non-holonomic and cable-driven manipulators, to biomechanical applications.

This issue is testimony of the enduring momentum and the active research towards promising future parallel manipulator, theoretical achievements, and fruitful applications as well. 